\title{
A SMOOTHNESS TEST FOR HIGHER CODIMENSIONS
}

\author{
JANKO BÖHM AND ANNE FRÜHBIS-KRÜGER
}

\begin{abstract}
Based on an idea in Hironaka's proof of resolution of singularities, we present an algorithmic smoothness test for algebraic varieties. The test is inherently parallel and does not involve the calculation of codimension-sized minors of the Jacobian matrix of the variety. We also describe a hybrid method which combines the new method with the Jacobian criterion, thus making use of the strengths of both approaches. We have implemented all algorithms in the computer algebra system SingulaR, and compare the different approaches with respect to timings and memory usage. The test examples originate from questions in algebraic geometry, where the use of the Jacobian criterion is impractical due to the number and size of the minors involved.
\end{abstract}

\section{INTRODUCTION}

In classical algebraic geometry, explicit constructions of algebraic varieties with prescribed properties play an important role, for example, for existence and unirationality results in moduli problems, see e.g. [24, 30. In many situations, the aim is to construct a smooth variety satisfying certain properties. For example, when considering a family of algebraic curves with a given Hilbert polynomial, the arithmetic genus of the curve is determined. The geometric genus, however, differs from the arithmetic genus by the delta invariant, which measures the singularities of the curve. Hence the presence of singularities affects the geometric genus, leading to a different topological type of the curve. Passing to the next dimension, recall that algebraic surfaces have been classified in the Enriques-Kodaira classification, see e.g. [2]. Especially for surfaces of general type, a full understanding of the moduli spaces and explicit constructions of canonical rings are still lacking. Although there are various techniques to construct surfaces with prescribed invariants, see for example [23, 24], the constraint of smoothness often requires testing and, in practice, this turns out to be a fundamental obstacle. It is thus of equal importance to have a test which is both fast in determining smoothness and non-smoothness.

The standard method for testing smoothness is the Jacobian criterion, (cf. any textbook on computational algebraic geometry, e.g. [19, Cor. 5.6.14]). Given an affine algebraic variety $X=V(I) \subseteq \mathbb{A}^{n}$ of codimension $c$ defined by an ideal $I=$ $\left\langle f_{1}, \ldots, f_{s}\right\rangle \subseteq k\left[x_{1}, \ldots, x_{n}\right]$ over an algebraically closed field $k$, we compute the dimension of the vanishing locus of the Jacobian ideal $J$, which is generated by $c \times c-$ minors of the Jacobian matrix $\left(\frac{\partial f_{i}}{\partial x_{j}}\right)$ on $X$. This can be done by computing a Gröbner basis of the ideal $I+J$. However, the number $\left(\begin{array}{l}n \\ c\end{array}\right) \cdot\left(\begin{array}{l}s \\ c\end{array}\right)$ of minors can be very large, and the Gröbner basis determines the complete scheme structure of the singular locus of $X$, which is not required to check smoothness. As a result, this approach will be rather inefficient and often even impractical.

In this paper, we describe an algorithm for determining smoothness, which is based on an idea from Hironaka's famous proof of resolution of singularities. The

2010 Mathematics Subject Classification. 14B05 (Primary), 68W10, 13P10, 32S05 (Secondary).

Key words and phrases. singularities, algebraic geometry, algorithmic smoothness test, Hironaka resolution of singularities, unprojection. 
(implicitly stated) termination criterion provides a smoothness criterion, which does not require computation of the $c \times c$-minors of the Jacobian matrix. The key idea behind this smoothness test is the fact that each non-singular variety is locally a complete intersection. That is, it can be covered by suitable open subsets, on each of which we truly see a complete intersection. Such a covering can be computed without too much effort and, in relevant examples, our approach significantly extends the limits of practicability of the Jacobian criterion. In addition, the construction is inherently parallel with only minimal communication overhead. In fact, application of the criterion can in some cases be faster than computing a single minor of the Jacobian matrix. Our algorithm is implemented in the library smoothtst.lib [6] for the computer algebra system Singular.

This paper is organized as follows: In Section 2, we extract the relevant part of Hironaka's smoothness criterion from the constructive, simplified desingularization approach of [12. In Section 3, we turn this criterion into an algorithm and then, in Section 4, refine it into a hybrid approach, which combines it with the use of the Jacobian criterion in smaller codimension. Section 5 introduces some settings and constructions from algebraic geometry where an efficient smoothness test is required, and presents explicit examples thereof. These are then used in Section 6 to compare our new approaches with the standard technique based on the Jacobian criterion.

\section{HironakA's Smoothness CRITERION}

In 1964, Hironaka proved existence of resolutions of singularities in characteristic zero [21]. He introduced standard bases to achieve this goal. Though his proof is non-constructive in certain parts, standard bases are by no means the only algorithmic considerations introduced there. The termination criterion, which he uses, provides a smoothness criterion that does not involve the computation of the ideal of codimension-sized minors of the Jacobian matrix.

For this article, we assume $k$ to be an algebraically closed field of characteristic zero unless explicitly stated otherwise. The general line of arguments is still valid, if we drop the condition on the field to be algebraically closed, but everything needs to be stated with significantly more care.

Definition 2.1. 21] Let $(X, p) \subset\left(\mathbb{A}_{k}^{n}, p\right)$ be a germ with defining ideal $I_{X, p} \subset$ $k\{\underline{x}\}:=k\left\{x_{1}, \ldots, x_{n}\right\}$ generated by $f_{1}, \ldots, f_{s}$, and assume that these power series form a standard basis of $I_{X, p}$ with respect to some local degree ordering. Assume further that the power series $f_{i}$ are sorted by increasing order. We then denote by $\nu^{*}(X, p)$ the sequence of orders of the $f_{i}$.

Making use of the sequence $\nu^{*}(X, p)$, we can formulate the termination condition in Hironaka's proof in the form of the following lemma, which is already implicitly present and used in [21]:

Lemma 2.2. The germ $(X, p) \subset\left(\mathbb{A}_{k}^{n}, p\right)$ is singular at $p$ if and only if

$$
\nu^{*}(X, p)>_{\text {lex }}(\underbrace{1, \ldots, 1}_{\operatorname{codim}(X)})
$$

with respect to the lexicographical ordering $>_{\text {lex }}$.

Of course, it is impractical to consider each point of a given variety separately, however this is a general problem when translating Hironaka's ideas into an algorithmic approach. Several groups have successfully tackled the problem of making Hironaka's approach completely constructive since the end of the 1980s (cf. [3], [12, 
[13]), and have created algorithmic approaches to desingularization. We will roughly follow here the approach of Bravo, Encinas and Villamayor, drop the condition of being a standard basis, and use the notion of the order of an ideal at a specific point $p$, which is precisely the first entry of $\nu^{*}(X, p)$. To make this notion accessible to computations, we state everything and do all calculations in affine charts, that is from the algebraic point of view, in polynomial rings. For the more general situation of an algebraic variety, the results can then be applied by means of a suitable cover of the variety by affine charts.

Definition 2.3. Let $W \subseteq \mathbb{A}_{k}^{n}$ be a smooth variety and let $I=\left\langle f_{1}, \ldots, f_{s}\right\rangle \subseteq k[\underline{x}]$ be a radical ideal defining a variety $X \subseteq W$. Then

$$
\operatorname{ord}_{p}(I):=\max \left\{m \in \mathbb{N} \mid I \subseteq \mathfrak{m}_{W, p}^{m}\right\} .
$$

Remark 2.4. Given an ideal $I$ as above and adapting [12] to our setting, we define the ideal sheaf $\Delta(I)$ as the ideal sheaf locally generated by $f_{1}, \ldots, f_{s}$ and all partial derivatives thereof with respect to a regular system of parameters of $W$. Then the locus of order at least two is precisely the vanishing locus of $\Delta(I)$, that is, the order of the ideal is (at most) one everywhere, if and only if $1 \in \Delta(I)_{w}$ for all $w \in W$.

Beware of a misunderstanding here: The above remark does not mean that there is one of the generators of $I$ which defines a smooth hypersurface, nor does it mean that we can use the same regular system of parameters everywhere. It only implies that, at each point of $X$, there is some generator defining a smooth hypersurface $W_{1} \subset W$ in some open neighborhood of the point (for an algorithmic treatment of the general case see [14, Section 4.2]).

Remark 2.5. Covering $X$ by affine charts corresponding to the complements of hypersurfaces, each containing the singular locus of one of the $f_{i}$, we can locally use $W \cap V\left(f_{i}\right)$ as the new ambient space $W_{1}$ and iterate our considerations 1 . If we ever encounter a maximal order exceeding one during this iteration - in any of the charts - we know that $X$ is singular.

The key idea behind the smoothness test is the fact that each non-singular variety is locally a complete intersection. Passing to a covering such that the variety is a complete intersection in each chart, all generators of the corresponding ideal are of order one.

The real difficulty in the process is the task of determining a suitable covering. Actually, the crucial point in turning this strategy into an algorithm is the choice of suitable regular systems of parameters; luckily this has already been made algorithmic in the more general case of the resolution of singularities in [14, [16], and [17]. Given such a system of parameters, it is then straight forward to perform the iteration and, hence, to test for smoothness. We give the details in the subsequent section.

\section{An Algorithmic Smoothness Test}

For the smoothness test described in this section, we assume that the input is an affine variety $X \subseteq W \subseteq \mathbb{A}_{k}^{n}$ such that the ambient variety $W$ is $\mathbb{A}_{k}^{n}$ at the start of the algorithm, that is, $I_{W}=\langle 0\rangle \subset k\left[x_{1}, \ldots, x_{n}\right]$. If we are dealing with more general

\footnotetext{
${ }^{1}$ For those readers who are familiar with algorithmic resolution of singularities, we would like to add two remarks: Due to the low order of the ideal and the absence of exceptional divisors from previous blow-ups, this is a specifically simple instance of the local existence of a hypersurface of maximal contact and a descent in ambient dimension by means of a coefficient ideal, see [12] or [14] for an implementable general construction.
} 
varieties, the smoothness test needs to be applied separately to each affine chart of the covering of the variety. In particular, a subvariety of projective space can be covered by the standard affine charts.

The top-level algorithm for our smoothness test is a rather simple recursion, for the details see Algorithm 1. This algorithm is just a reformulation of the ideas of Section 2 and, of course, nothing but a hollow shell without the two workhorses described in Algorithms 2 and 3, which we will now discuss in detail.

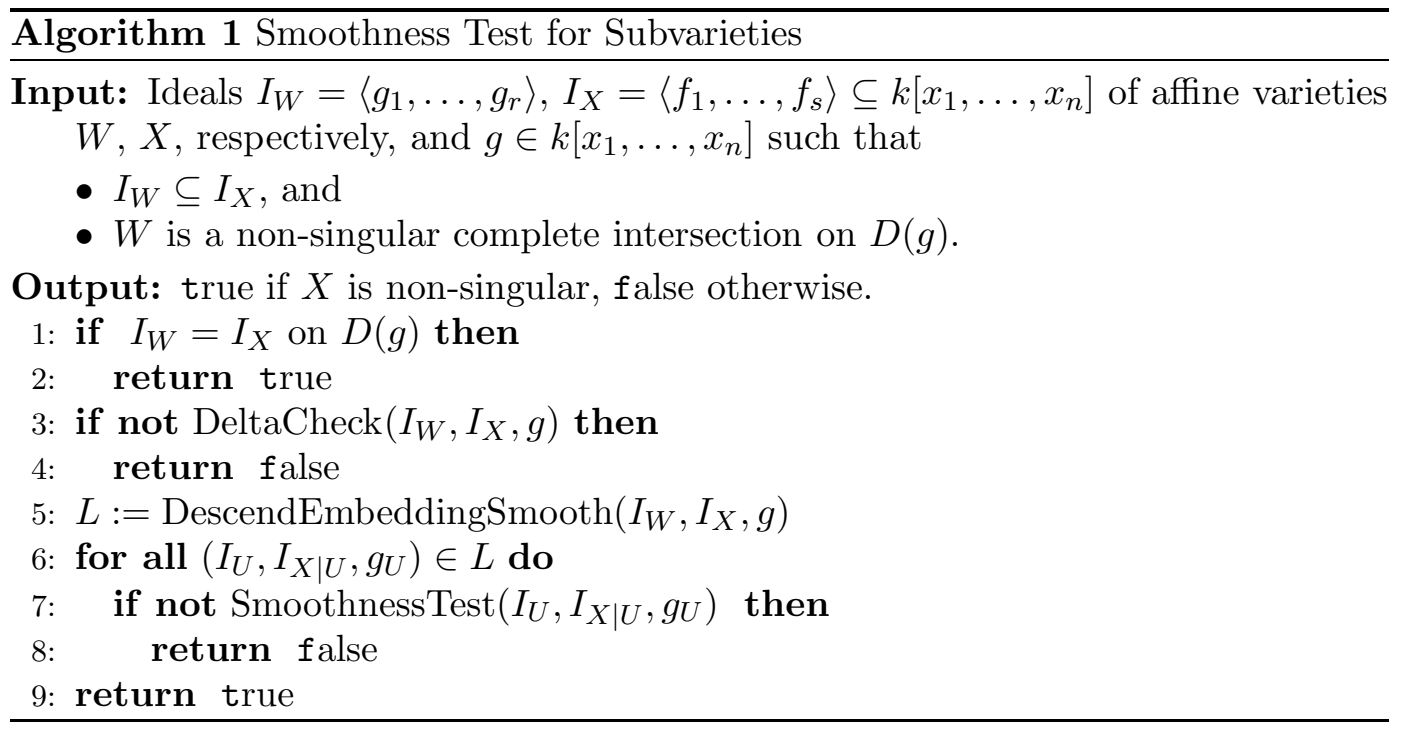

Notation 3.1. For stating the algorithms of this section, we adhere to standard notation of algebraic geometry denoting the complement of the vanishing locus of a polynomial $g \in k\left[x_{1}, \ldots, x_{n}\right]$ by $D(g)$. The equality test for $I_{W} \subseteq I_{X}$ on $D(g)$ then amounts to checking whether $g \cdot I_{X}$ is contained in $\sqrt{I_{W}}=I_{W}$ (where the last equality holds, because $W$ is smooth).

Algorithm 2, DeltaCheck, is designed to detect non-singularity as soon as possible with a minimal amount of time and memory consumption. To this end, it first treats the 'lucky' trivial case, where the ambient space $W$ is just $\mathbb{A}_{k}^{n}=\operatorname{Spec}\left(k\left[x_{1}, \ldots, x_{n}\right]\right)$, and the regular system of parameters is hence given by the variables. If a regular system of parameters needs to be determined, we can make use of the smoothness of the ambient space $W$, which implies that at each point of $W \cap D(g)$ at least one of the $r \times r$ minors of the Jacobian matrix of $I_{W}=\left\langle g_{1}, \ldots, g_{r}\right\rangle$ is non-zero. Thus, the complements of these minors give rise to an open covering of $W \cap D(g)$, and, on each open set, the columns which are unused with respect to the invertible minor lead to a regular system of parameters. But, since coordinate changes are expensive, the explicit passage to the new coordinates (and back) is avoided by an appropriate modification of the derivatives (see line 14). Eventually, we only need to check whether $q g \in \sqrt{C_{M}}$, because this amounts to the same as checking whether $V\left(C_{M}\right)$ is contained in the union of the hypersurfaces $V(g)$ and $V(q)$.

Remark 3.2. It is, in general, not a good idea to run this computation for all $\left(\begin{array}{l}n \\ r\end{array}\right)$ minors. Instead, the computation should stop as soon as all of $W \cap D(g)$ is covered by the open sets. This explains the second termination condition of the main loop. As an enhancement, it is possible to further reduce the number of charts by expressing $g$ in terms of the minors of $\operatorname{Jac}\left(I_{W}\right)$ and then to only consider those minors appearing. 


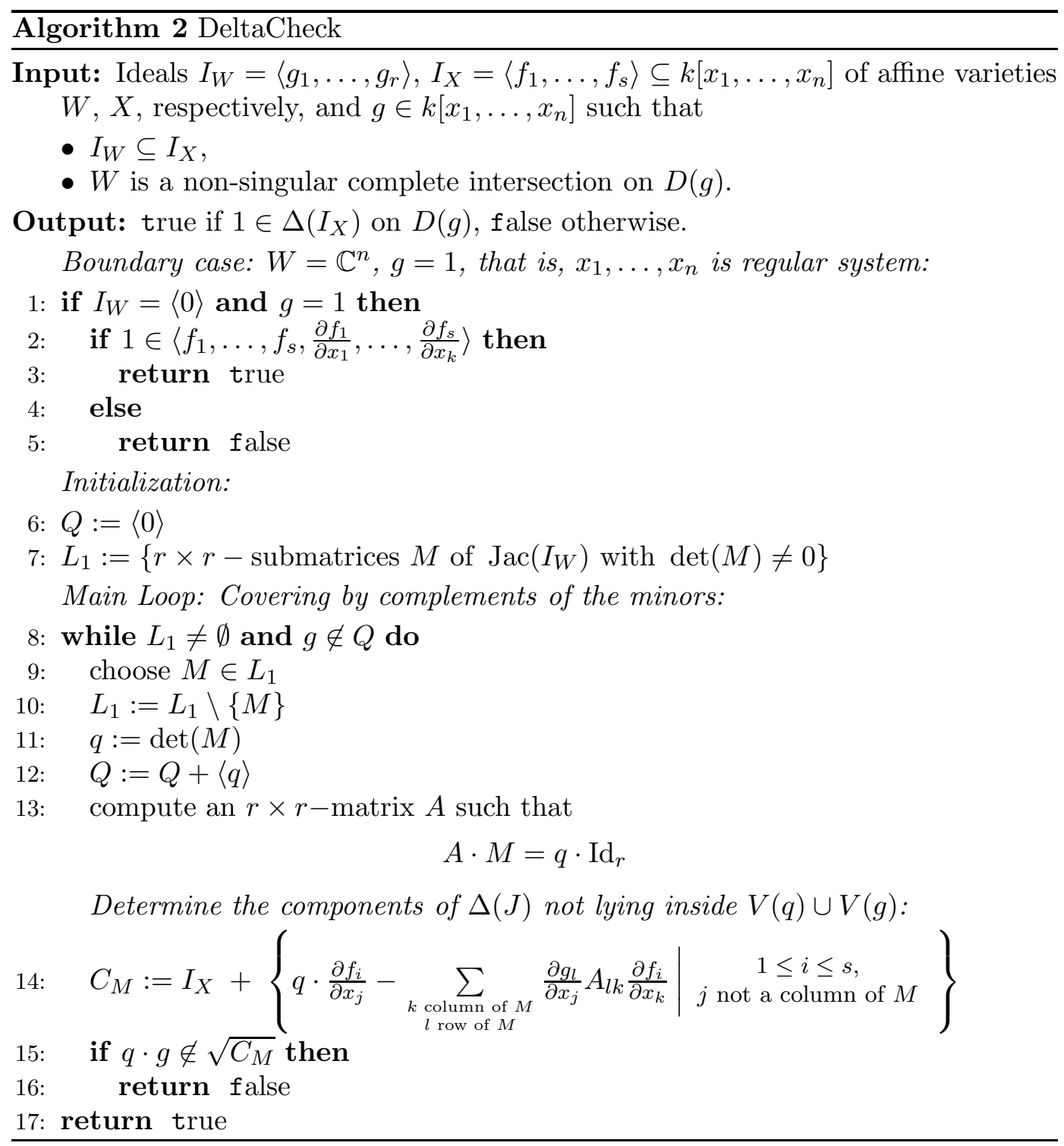

Expressing the element $g$ in terms of the generators of an ideal can easily be implemented in Singular: the command lift performs this operation on the basis of a single standard basis computation. Thus the previously considered enhancement is actually a trade-off, as it avoids redundant calculations at the cost of an additional standard basis computation.

The general idea of the Algorithm 3, DescendEmbeddingSmooth, is the one described in its lines 1 to 4 . Find an element of $I_{X}$, which defines a non-singular hypersurface in $W \cap D(g)$, use this hypersurface as a new ambient space and return the new triple. Unfortunately, the situation is more complicated, as, in general, such a hypersurface does not exist globally. An open covering is thus required to find such a hypersurface on each open subset (as already outlined in the previous section). On the other hand, we know that the maximal order of $I_{X}$ at each point of $W \cap D(g)$ is at most one, which implies that the intersection of the singular loci of the hypersurfaces $V\left(f_{i}\right) \cap D(g)$ in $W \cap D(g)$ is empty. When expressing $g$, which of course is a unit on $D(g)$, in terms of the generators of the ideals of the singular loci, only some of the generators actually appear in the corresponding $k\left[x_{1}, \ldots, x_{n}\right]$-linear combination with non-zero coefficients. These are the polynomials, for which the 


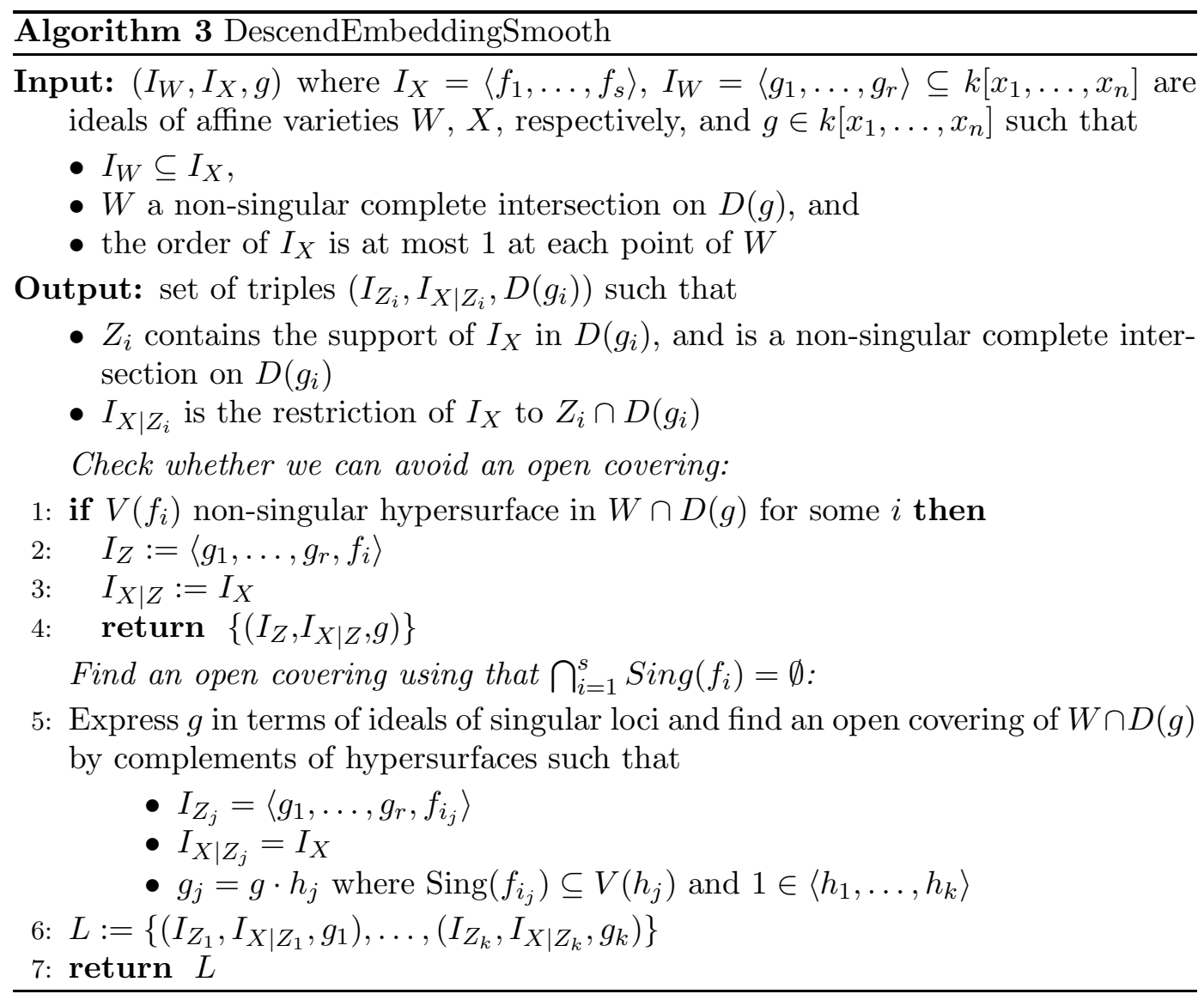

complements of the vanishing loci define the desired open covering. As soon as this covering has been found, we can proceed as in steps 1 to 4 for each of the open sets.

Note that, to apply Algorithm 3 in Algorithm 1, we actually need that $Z_{i}$ contains the locus of maximal order of $I_{X}$ on $D(g)$. But at this point we already know that the maximal order is one, whence the locus of maximal order is just the vanishing locus of $I_{X}$. Note also that $I_{X \mid Z_{i}}$ is the coefficient ideal of $I_{X}$ with respect to $Z_{i}$ since the maximal order of $I_{X}$ is one.

If we assume that Algorithm 1 was initially called with $W=\mathbb{A}_{k}^{n}$, the construction at each level of descent in ambient dimension provides an ambient space which is a complete intersection on $D(g)$. If the top-level ambient space, that is, the original input, is not a complete intersection, it may be necessary to find an open covering such that this smooth ambient space, which locally is a complete intersection, is specified by a complete intersection on each open set itself, before the smoothness test for $X \subseteq W$ can be applied. For an affine variety $W$, this can be achieved by applying Algorithm 3 to $W \subseteq \mathbb{A}_{k}^{n}$ codimension times.

Remark 3.3. The above Algorithms 1 and 2 are inherently parallel, as both rely on open coverings and the computations in the respective open sets are independent of each other. More precisely, the main loop of Algorithm 1, in which the recursion occurs, can be distributed to multiple cores and the computation of $\Delta\left(I_{X}\right)$ on the different sets $D(q) \cap D(g)$ also readily allows parallelization.

Due to the boolean return type, the total communication overhead of parallelizing one of these steps originates from handing data to the routine, not from returning a result. The embedding dimension, on the other hand, provides an upper bound 
for the depth of the recursion. In combination, this implies that the communication overhead for a fully parallel approach will be insignificant compared to the benefits in computation time.

Remark 3.4. For practical purposes, in line 1 of Algorithm 3, it is worth to try a general linear combination of $f_{1}, \ldots, f_{s}$ as well, before passing to an open cover.

Remark 3.5. There is one potential drawback to the approach outlined in this section: In comparison to the standard approach of using the Jacobian criterion, the presented algorithms do not only rely on arithmetic operations on a fixed set of polynomials and one subsequent standard basis computation. They need, for example, standard bases computations for the radical membership tests. This does not only make them more sensitive to intermediate coefficient swell, but also introduces imponderabilities with regard to the complexity of a given example due to the well known difference between average and worst-case complexity of computing standard bases.

Remark 3.6. Up to this point, we always assumed that char $k=0$. This may seem to be an obvious restriction, given that we are extracting the approach from the proof of resolution of singularities, which is a famous open problem in positive characteristic. Considering the obstacles for generalizing Hironaka's approach to positive characteristic a bit more closely (as was done, e.g., in [20], and, with focus on constructing examples, also in [15]), it is obvious that all of the unresolved complications arise during the sequence of blow-ups, not within the smoothness-test. So this issue does not pose a serious obstruction to using the above algorithms over a perfect field in positive characteristic in order to check smoothness of a reduced variety $X \subseteq \mathbb{A}_{k}^{n}$. One could, thus, also envision the use of a modular approach to the characteristic zero problem.

\section{A Hybrid Approach}

The main drawback of the classical smoothness test by means of the Jacobian criterion is the high number of minors to be computed. It may therefore not only turn out to be time consuming, but also requires huge amounts of memory. In practice, the latter is the more restricting aspect, as it may cause a premature termination of the computation due to lack of memory either while computing the minors or while determining a Gröbner basis of the Jacobian ideal. The new approach can also lead to involved Gröbner basis computations - in particular, if the polynomials defining the open sets $D(g)$ are of high degree and contain many terms.

So both approaches have rather obvious drawbacks, but the one of the classical approach is most relevant if the codimension of the given variety is large and the variety is far from being a complete intersection. For the new approach, however, the main drawback does not become significant, before several descents in ambient dimension have been applied. Each descent in ambient dimension lowers the codimension of the variety. Hence, it makes sense to use the new algorithm as a preparation step for applying the Jacobian criterion in a smaller codimension.

In Algorithm 1, this hybrid approach can easily be introduced by adding a new input parameter controlling the depth of the descent in ambient dimension and checking between lines 4 and 5 , whether the maximal desired number of descents has been made. If so, we call Algorithm 4 to check smoothness by the Jacobian criterion and return its result.

Algorithm 4, which applies the Jacobian criterion in a relative situation, is again a hybrid between two algorithms that we have seen before. The computation of a regular system of parameters (possibly after passing to an open covering) and the 


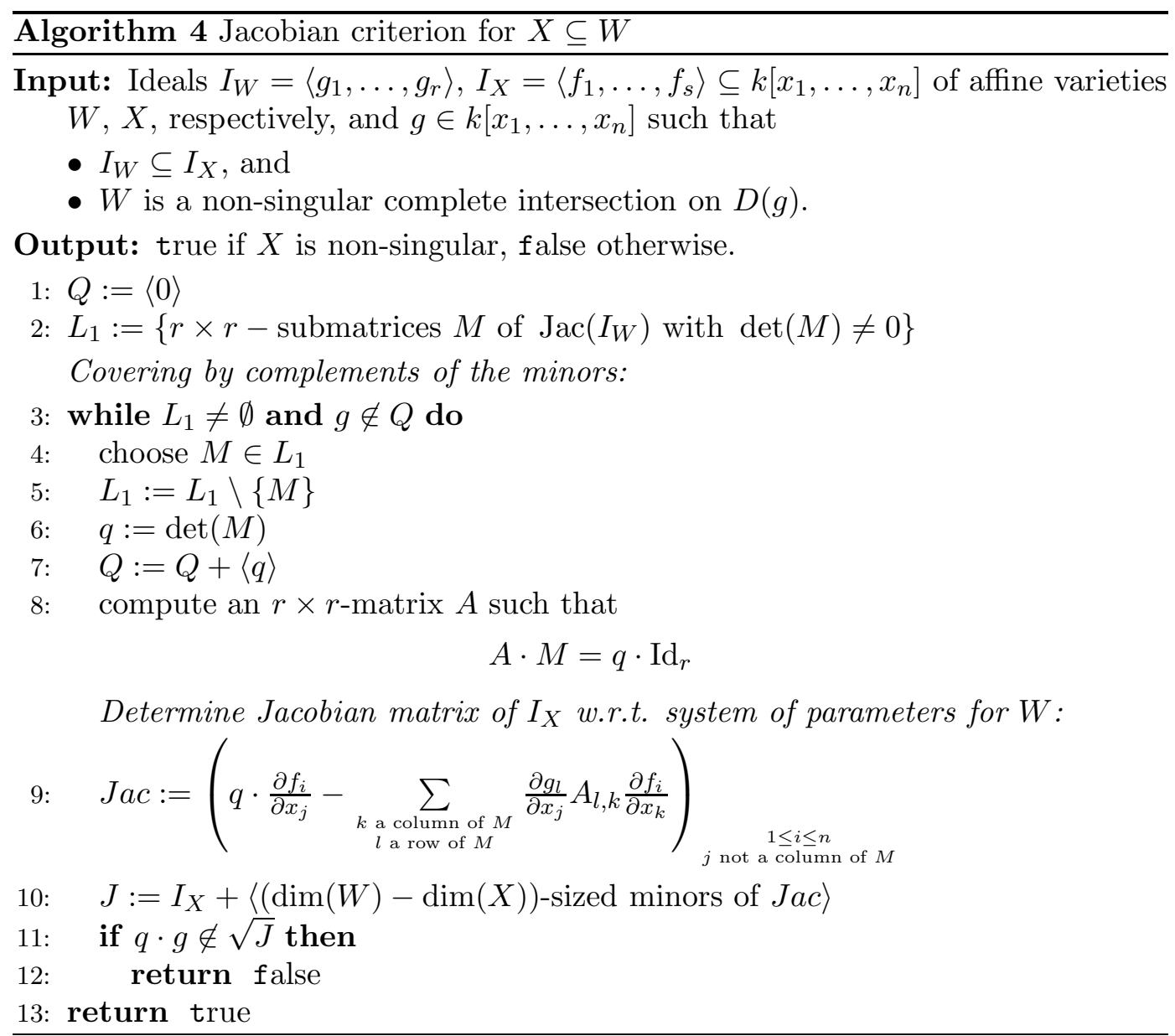

computation of the Jacobian matrix with respect to this system coincide precisely with the respective steps of Algorithm 2. The remaining steps are a straight forward application of the Jacobian criterion to the before-computed Jacobian matrix.

This hybrid approach inherits the inherently parallel structure of the new algorithm, lowers the combinatorial complexity of the Jacobian criterion by applying several descents of ambient dimension beforehand, and avoids - to a certain extent - the imponderabilities of the large number of radical membership tests arising from covering and descending as many times as the codimension requires. In this way, we obtain a viable divide-and-conquer approach for parallelizing the Jacobian criterion.

\section{Applichtions in Algebraic Geometry}

In this section, we describe several constructions from algebraic geometry, which all aim at obtaining varieties with specific properties. All resulting ideals are specified as examples in our library smoothtst.lib. We use these examples in Section 6 to study the performance of our smoothness test in realistic usage scenarios.

The constructions use the technique of Kustin-Miller unprojection to produce sequences of birationally equivalent varieties. Unprojection theory was introduced by A. Kustin, M. Miller, M. Reid, and S. Papadakis (see [22, 29, 27, 26]) to construct Gorenstein rings of high codimension and to act as a substitute for a structure theorem for Gorenstein rings of codimension $\geq 4$. We give a short outline of the fundamental construction used in the examples, for details see [9]. This construction is implemented in the package KustinMiller [10] for the computer algebra system MaCAUlay2 [18]. 
Suppose that $R$ is a positively graded polynomial ring over a field. Given two homogeneous ideals $I \subset J \subset R$ defining Gorenstein rings $R / I$ and $R / J$ such that $\operatorname{dim} R / I=\operatorname{dim} R / J+1$, that is, $V(J)$ is codimension 1 subvariety of $V(I)$, we will construct a new Gorenstein ring, the unprojection ring. Geometrically the construction corresponds to the contraction of the subvariety $V(J) \subset V(I)$. By [11, Proposition 3.6.11] there are $k_{1}, k_{2} \in \mathbb{Z}$ such that $\omega_{R / I}=R / I\left(k_{1}\right)$ and $\omega_{R / J}=$ $R / J\left(k_{2}\right)$. Suppose that $k_{1}>k_{2}$. This implies that the unprojection ring is also positively graded.

Definition 5.1. 27] Let $\iota: J / I \rightarrow R / I$ be the inclusion morphism and let $\phi$ : $J / I \rightarrow R / I$ be a homomorphism of degree $k_{1}-k_{2}$ such that $\operatorname{Hom}_{R / I}(J / I, R / I)$ is generated as an $R / I$-module by $\iota$ and $\phi$. The Kustin-Miller unprojection ring defined by $\phi$ is the graded algebra

$$
(R / I)[T] / U \quad \text { where } \quad U=\langle T u-\phi(u) \mid u \in J / I\rangle,
$$

and $\operatorname{deg}(T):=k_{1}-k_{2}$.

Note that $(R / I)[T] / U \cong R[T] / \widetilde{U}$, where $\widetilde{U}$ is the inverse image of $U$ under the natural map $R[T] \rightarrow(R / I)[T]$.

Proposition 5.2. 22, 27] The R-algebra $R[T] / U$ is Gorenstein and, up to isomorphism, independent of the choice of $\phi$.

Example 5.3. Consider a cubic surface in $\mathbb{P}^{3}$ with six disjoint lines. Such a surface corresponds to the blow-up of $\mathbb{P}^{2}$ in six points (for an implementation of this construction, see the MACAULAY2 package clebsch.m2 7]). Applying the Kustin-Miller unprojection construction to the ideal $J$ of such a line corresponds to the Castelnuovo blow-down of the line and results in a Del Pezzo surface in $\mathbb{P}^{4}$ of degree 4 . By iterating this construction, we obtain a sequence of Del Pezzo surfaces of degree $2+c$ and codimension $c$ in $\mathbb{P}^{2+c}$ for $c=1, \ldots, 7$. See [25, Section 4.1] for more details.

Example 5.4. Consider the codimension 2 complete intersection threefold $X_{2} \subset \mathbb{P}^{5}$ defined by

$$
I=\left\langle x_{1} x_{3}-y_{1} y_{2}, x_{2} x_{4}-y_{1} y_{2}\right\rangle
$$

and the ideals $J_{1}=\left\langle y_{1}, x_{1}, x_{2}\right\rangle, J_{2}=\left\langle y_{2}, x_{2}, x_{3}\right\rangle, J_{3}=\left\langle y_{1}, x_{3}, x_{4}\right\rangle$, and $J_{4}=$ $\left\langle y_{1}, x_{4}, x_{1}\right\rangle$. Applying the Kustin-Miller construction iteratively for the ideal $J_{1}$ introducing the new variable $T_{1}$, for $J_{2}+\left\langle T_{1}\right\rangle$ introducing $T_{2}$, for $J_{3}+\left\langle T_{1}, T_{2}\right\rangle$ introducing $T_{3}$, and for $J_{4}+\left\langle T_{1}, T_{2}, T_{3}\right\rangle$ introducing $T_{4}$, we obtain a sequence $X_{2}, \ldots, X_{6}$ of threefolds of codimensions $2, \ldots, 6$. Note that $X_{2}, X_{3}, X_{4}$ are singular, whereas $X_{5}$ and $X_{6}$ are smooth.

Example 5.5. Let $R=k\left[x_{1}, \ldots, x_{n}\right]$ be a polynomial ring over a field $k$ and let $I_{d}(R)$ be the Stanley-Reisner ideal of the boundary complex of the cyclic polytope of dimension $d$ with vertices $x_{1}, \ldots, x_{n}$. (Recall that the Stanley-Reisner ideal is the monomial ideal generated by the non-faces of the complex.) As shown in [8], KustinMiller unprojection yields a recursion for the ideals $I_{d}(R)$ : For $d$ even, apply the construction with $T=x_{n}$ to $I=I_{d}\left(k\left[x_{1}, \ldots, x_{n-1}\right]\right)$ and $J=I_{d-2}\left(k\left[z, x_{2}, \ldots, x_{n-2}\right]\right)$ considered as ideals in $k\left[z, x_{1}, \ldots, x_{n-1}\right]$ and quotient by $\langle z\rangle$. For $d$ odd one can proceed in a similar way, for details see [8, Section 4]. Note that the varieties defined by the ideals $I_{d}(R)$ are not smooth. 


\section{EfFiciency And Timings}

The Hironaka style smoothness test as presented in Section 3 together with the hybrid variant discussed in Section 4 are implemented in the SINGULAR library smoothtst.lib, see [6]. In this section, we compare the performance of these approaches to the smoothness test given by the Jacobian criterion (see Table1, columns smoothtst, hybrid, and Jacobian).

Note that, when computing minors of the Jacobian matrix of $X=V(I) \subset \mathbb{A}^{n}$, we consider all results of arithmetic operations in $k\left[x_{1}, \ldots, x_{n}\right] / I$, that is, we reduce modulo $I$. This leads to a significant improvement of the performance of the Jacobian criterion. In the hybrid approach, we exemplarily give timings for descending to codimension 2, that is, applying the Jacobian criterion for $2 \times 2-$ minors.

As discussed above, the communication overhead of a parallel version of the algorithm depends only on the depth of the recursion tree generated in the course of the algorithm. This depth is bounded by the embedding dimension. Moreover, the return type of the recursion is of type boolean. As a result, the algorithm has basically zero communication overhead. Hence, to show the full potential of the algorithm with respect to massively parallel computations, we give simulated parallel timings even if the number of processes exceeds the number of cores available on our machine (see subcolumns time for sequential and parallel for parallel timings).

All timings are in seconds on an Intel Xeon E5-2690 machine with 32 cores, 2.9 $\mathrm{GHz}$, and $192 \mathrm{~GB}$ of RAM running a Linux operating system. In addition to timings, we also do a comparison with respect to memory usage by indicating the maximum amount of memory used by SingULAR in megabytes when running the algorithms on a single core (subcolumns mem). Dashes indicates that the respective computation did not finish within 10000 seconds or used more than 20GB of RAM.

In the table we also indicate, whether the specific example is smooth (column smooth). We have verified that for all examples, where the Jacobian criterion finishes or where the presence/absence of singularities is a known from theoretical considerations, our implementation of the new algorithm comes to the same result.

TABle 1. Timings and Memory Usage

\begin{tabular}{l|c|c|c|c|c|c|c|c|c|} 
& & \multicolumn{3}{|c|}{ smoothtst } & \multicolumn{3}{|c|}{ hybrid } & \multicolumn{2}{c|}{ Jacobian } \\
& smooth & time & parallel & mem & time & parallel & mem & time & mem \\
\hline \hline $\mathcal{I}_{1}(6)$ & yes & 0.24 & 0.07 & 0.22 & 0.18 & 0.05 & 0.22 & 2.5 & 34 \\
\hline $\mathcal{I}_{1}(7)$ & yes & 0.60 & 0.17 & 0.24 & 0.35 & 0.10 & 0.22 & 310 & 1300 \\
\hline $\mathcal{I}_{1}(8)$ & yes & 0.86 & 0.22 & 0.32 & 0.64 & 0.15 & 0.23 & - & $>20000$ \\
\hline \hline $\mathcal{I}_{2}(3)$ & yes & 0.22 & 0.04 & 0.14 & 0.08 & 0.02 & 0.14 & 0.05 & 4.2 \\
\hline $\mathcal{I}_{2}(4)$ & yes & 160 & 9.1 & 27 & 40 & 4.9 & 190 & 15 & 450 \\
\hline $\mathcal{I}_{2}(5)$ & yes & - & - & - & 1200 & 14 & 510 & 4000 & 16000 \\
\hline \hline $\mathcal{I}_{3}(4)$ & no & 0.30 & 0.05 & 0.22 & 0.15 & 0.03 & 0.22 & 1.0 & 8.6 \\
\hline $\mathcal{I}_{3}(5)$ & yes & 0.72 & 0.10 & 0.22 & 0.38 & 0.07 & 0.22 & 110 & 300 \\
\hline $\mathcal{I}_{3}(6)$ & yes & 1.3 & 0.18 & 0.22 & 0.83 & 0.11 & 0.22 & 2500 & 2300 \\
\hline \hline $\mathcal{I}_{4}(6,3)$ & no & 0.02 & 0.01 & 0.14 & 0.02 & 0.01 & 0.14 & 3.1 & 34 \\
\hline $\mathcal{I}_{4}(7,3)$ & no & 0.04 & 0.02 & 0.14 & 0.04 & 0.01 & 0.14 & 1600 & 4000 \\
\hline $\mathcal{I}_{4}(7,4)$ & no & 0.10 & 0.02 & 0.14 & 0.10 & 0.02 & 0.14 & 4300 & 4000 \\
\hline
\end{tabular}

We now turn to the specific examples. In the table, we first consider curves: For $d \geq 1$, let $\mathcal{I}_{1}(d) \in \mathbb{Q}\left[x_{0}, \ldots, x_{d}\right]$ be the ideal generated by the $2 \times 2$-minors of the 
matrix

$$
\left(\begin{array}{ccc}
x_{0} & \ldots & x_{d-1} \\
x_{1} & \ldots & x_{d}
\end{array}\right)
$$

defining a rational normal curve of degree $d$ in $\mathbb{P}^{d}$. Note that these curves are smooth.

With respect to surfaces, we consider the following examples: The ideals $\mathcal{I}_{2}(c) \in$ $\mathbb{F}_{103}\left[x_{0}, \ldots, x_{3}, s_{1}, \ldots, s_{c-1}\right]$ for $c=2, \ldots, 7$ define del Pezzo surfaces of degree $2+c$ of codimension $c$ in $\mathbb{P}^{2+c}$, as constructed in Example 5.3. The ideals $\mathcal{I}_{3}(m)$ for $m=2, \ldots, 6$ correspond to the unprojection sequence from Example 5.4 defined over the rationals.

Finally the ideals $\mathcal{I}_{4}(d, n)$ define the Stanley-Reisner rings of the cyclic polytopes of dimension $d$ with $n$ vertices from Example 5.5, after a random linear coordinate change over the rationals with coefficients of bitlength 4 .

Remark 6.1. A parallel implementation of the Jacobian criterion is in the process of development. The results obtained so far show that, although the step computing the minors can easily be parallelized, combining the results of the cores in use into a single ideal leads to a significant communication overhead 2 Parallelization of Gröbner basis computations is a topic of current research, but still has no general solution. When computing over the rationals, one approach to parallelization is to consider modular methods, see [1, 4]. The conclusion is that the Jacobian criterion will benefit much less from parallel computations than our algorithm, hence we do not give any timings with regard to a parallel Jacobian criterion.

Note however, that in many applications, for example when computing the normalization of an affine algebra as in [5], knowledge of the Jacobian ideal will be required. Thus, even taking the new smoothness test into account, an efficient parallel approach for the computation of the Jacobian ideal is of key importance.

To summarize, we observe that the new algorithm is significantly faster than the Jacobian criterion in those examples in consideration, where the variety is singular, even when only running on a single core. For testing smoothness of non-singular varieties, it can be slow if the Groebner basis computations involved are complicated, or a very large number of charts has to be considered. Even in these cases, the memory requirements are significantly lower than in the Jacobian criterion approach.

The algorithm can benefit to a great extent from parallel and massive parallel computations, which are of increasing importance considering current and future hardware. As outlined in Section 4, this can also be exploited to implement a divideand-conquer strategy by first doing several descends of ambient dimension using the new approach and then applying the Jacobian criterion in each chart. Combining the advantages of both approaches, this hybrid algorithm eventually turns out to be the most successful in examples in consideration.

Acknowledgements. We would like to thank Mirko Rahn, Lukas Ristau, Stavros Papadakis, Bernd Schober, and Yue Ren for helpful discussions.

\section{REFERENCES}

[1] Arnold, E. A.: Modular algorithms for computing Gröbner bases, Journal of Symbolic Computation 35, 403-419 (2003).

\footnotetext{
${ }^{2}$ The parallelization framework for this massive parallel approach is GPI-space, a low latency communication library and runtime system for scalable real-time parallel applications developed at the Fraunhofer ITWM [28. Its use for various computational settings in algebraic geometry is subject to an ongoing joint project between ITWM and the Singular development team.
} 
[2] Barth, W. P.; Hulek, K.; Peters, Chris A. M.; Van de Ven, A.: Compact Complex Surfaces, Ergebnisse der Mathematik und ihrer Grenzgebiete. 3. Folge. 4, Springer (2004).

[3] Bierstone, E.; Milman, P.: Canonical Desingularization in Characteristic Zero by Blowing up the Maximum Strata of a Local Invariant, Invent.Math. 128 (1997), 207-302

[4] Böhm, J.; Decker, W.; Fieker, C.; Pfister, G.: The use of bad primes in rational reconstruction. Math. Comput. 84 (296) (2015).

[5] Böhm, J.; Decker, W.; Laplagne, S.; Pfister, G.; Steenpaß, A.; Steidel, S.: Parallel Algorithms for Normalization. J. Symbolic Comput. 51 (2013), 99-114.

[6] Böhm, J.; Frühbis-Krüger, A.: smoothtst.lib - A Singular 4-0-2 library for determining smoothness of algebraic varieties. Singular distribution, http://www.singular.uni-kl.de.

[7] Böhm, J.; Marais, M.: clebsch.m2 - Constructing cubic surfaces and lines thereon, Macaulay2 package (2014).

[8] Böhm J.; Papadakis S.: On the structure of Stanley-Reisner rings associated to cyclic polytopes, Osaka J. Math. 49 (2012), no. 1, 81-100.

[9] Böhm J.; Papadakis S.: Implementing the Kustin-Miller complex construction, J. Softw. Algebra Geom. 4 (2012), 6-11.

[10] Böhm J.; Papadakis S.: KustinMiller - The Kustin-Miller complex construction and resolutions of Gorenstein rings, Macaulay2 package (2012).

[11] Bruns W.; Herzog J.: Cohen-Macaulay Rings, revised edition, Cambridge Studies in Advanced Mathematics 39, Cambridge University Press, Cambridge, 1998.

[12] Bravo, A. M.; Encinas, S.; Villamayor, O.: A Simplified Proof of Desingularization and Applications. Rev. Mat. Iberoamericana 21 (2005), no. 2, 349-458.

[13] Encinas, S.; Hauser, H.: Strong resolution of singularities in characteristic zero, Comment. Math. Helv. 77 (2002), 821-845.

[14] Frühbis-Krüger, A.: Computational Aspects of Singularities, in J.-P. Brasselet, J.Damon et al.: Singularities in Geometry and Topology, World Scientific Publishing (2007), 253-327.

[15] Frühbis-Krüger,A.: A short note on Hauser's Kangaroo phenomena and weak maximal contact in higher dimensions, J. Sing. 2 (2010), 128-142

[16] Frühbis-Krüger,A.: Desingularization in Computational Applications and Experiments, in Ellwood, Hauser et al.(Eds): The resolution of Singular Algebraic Varieties, Clay Mathematics Proceedings 20 (2014), 269-284

[17] Frühbis-Krüger, A.; Pfister, G.: resolve.lib - A Singular 4-0-2 library for the resolution of singularities. Singular distribution, http://www.singular.uni-kl.de

[18] Grayson, D.; Stillman, M.: Macaulay2, a software system for research in algebraic geometry, available at http://www.math.uiuc.edu/Macaulay2/

[19] Greuel, G.-M.; Pfister, G.: A Singular Introduction to Commutative Algebra. Springer (2008).

[20] Hauser, H.: Why the characteristic zero proof of resolution fails in positive characteristic Manuscript 2003, available at http://www.hh.hauser.cc

[21] Hironaka, H.: Resolution of singularities of an algebraic variety over a field of characteristic zero. I, II. Ann. of Math. (2) $\mathbf{7 9}$ (1964), 109203, 205326.

[22] Kustin, A.; Miller, M.: Constructing big Gorenstein ideals from small ones, J. Algebra 85 (1983), 303-322.

[23] Mendes Lopes, M.; Pardini, R.: Numerical Campedelli surfaces with fundamental group of order 9, J. Eur. Math. Soc. 10 (2008), no. 2, 457476.

[24] Neves, J.; Papadakis, S.: A construction of numerical Campedelli surfaces with ZZ/6 torsion, Trans. Amer. Math. Soc. 361 (2009), 4999-5021.

[25] Neves, J.; Papadakis, S.: Parallel Kustin-Miller unprojection with an application to Calabi-Yau geometry, Proc. London Math. Soc. 106 (2013), 203-223.

[26] Papadakis, S.: Kustin-Miller unprojection with complexes, J. Algebraic Geom. 13 (2004), 249268.

[27] Papadakis, S.; Reid, M.: Kustin-Miller unprojection without complexes, J. Algebraic Geom. 13 (2004), 563-577.

[28] Pfreundt, F.-J. ; Rahn, M.; et al.: GPI-space, Fraunhofer ITWM Kaiserslautern, http://www.gpi-space.de/

[29] Reid, M.: Graded rings and birational geometry, in Proc. of Algebraic Geometry Symposium (K. Ohno, ed.), Kinosaki, Oct. 2000, pp. 1-72, available at http://www.warwick.ac.uk/ masda/3folds

[30] Schreyer, F.-O.: Computer aided unirationality proofs for moduli spaces, Handbook of Moduli, Volume III (2013), 257-280. 
Department of Mathematics, University of Kaiserslautern, ERWin-Schrödinger-Str., 67663 Kaiserslautern, Germany

E-mail address: boehm@mathematik.uni-kl.de

Institut für algebraische Geometrie, Leibniz Universität Hannover, Welfengarten 1, 30167 Hannover, Germany

E-mail address: anne@math.uni-hannover.de 